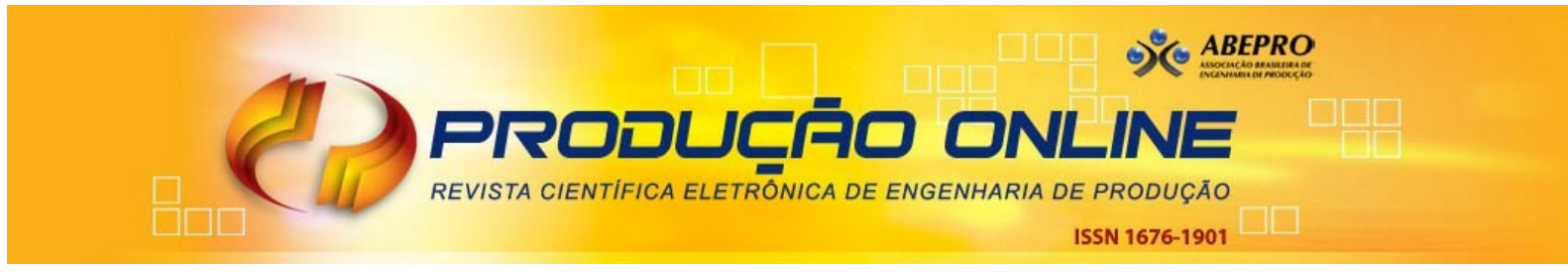

\title{
AVALIAÇÃO DE DESEMPENHO: PROCESSO DE REVISÃO SISTÊMICA DE LITERATURA INTERNACIONAL
}

\section{PERFORMANCE EVALUATION: PROCEDURE OF THE SYSTEMIC REVIEW OF INTERNATIONAL LITERATURE}

\author{
Fabricia Silva da Rosa* E-mail: fabriciasrosa@hotmail.com \\ Sandra Rolim Ensslin** E-mail: sensslin@gmail.com \\ Leonardo Ensslin** E-mail: leonardoensslin@gmail.com \\ *Univesitat de Valencia, ES \\ ** Universidade Federal de Santa Catarina (UFSC), Florianópolis, SC
}

\begin{abstract}
Resumo: Avaliação de Desempenho pode ser utilizada em diversos contextos para avaliar pessoas e organizações com o objetivo de auxiliar em processos decisórios, sendo amplamente difundida no meio científico. Contudo, considera-se importante verificar como o assunto é estudado. Nesse contexto, surge a pergunta de pesquisa que orienta esta investigação: "Quais são os critérios que devem ser considerados em um processo estruturado que pretende identificar o referencial teórico com validade científica e promover revisão sistêmica que permita identificar potencialidades e oportunidades dos modelos de Avaliação de Desempenho?" Assim, objetiva-se identificar as potencialidades e oportunidades de uma amostra de artigos. Para isso, utiliza-se um processo estruturado de revisão de literatura que abrange três etapas: (i) seleção de artigos, (ii) análise bibliométrica dos artigos selecionados e (iii) revisão sistêmica. Com base nesses resultados, se verifica as oportunidades para promover aperfeiçoamento nos processos de Avaliação de Desempenho e sugere aplicação de modelo de Apoio à Decisão sobre contextos singulares.
\end{abstract}

Palavras-chave: Avaliação de Desempenho. Processo Estruturado de Revisão de Literatura. Revisão Sistêmica.

\begin{abstract}
Performance assessment can be used in different contexts to assess individuals and organizations, aiming to assist decision making. It is widespread in the scientific community, however it is important to check how the subject is studied. In this context, there is the research question: What criteria should be considered in a structured process that you want to identify a theoretical scientific validity, and promote systemic review identifying potential opportunities and models performance assessment? Thus, the objective is to identify strengths and opportunities of a sample of articles. To do this, it uses a structured process of literature review which covers three stages: (i) selection of articles, (ii) bibliometric analysis of selected articles, (iii) systemic review. Based on these results the research infers about the opportunities to promote improvement in the processes of performance evaluation, and suggests the application of decision support model for particular contexts.
\end{abstract}

Keywords: Performance Assessment. Structured Process for Literature Review. Systemic Review.

\section{INTRODUÇÃO}

Apesar do crescente interesse do mundo empresarial pelo desenvolvimento de novas visões científicas a fim de promover análises que contemplem a avaliação do desempenho de forma singular ao ambiente que se propõe ajudar, 
ainda existe um reduzido número de aplicações nesse assunto (WILLIAMS, 2005; SHENHAR; DVIR, 2007). Algum sucesso nesse sentido tem sido alcançado nos trabalhos de Dvir et al. (1998), Lacerda (2009), Dvir e Lechler (2004), Shenhar e Dvir (2007), Tukel e Rom (2001) e Williams (2005). Uma visão que integre avaliação do sucesso a seus aspectos operacionais, segundo Dvir et al. (1998), Shenhar (2004) e Shenhar e Dvir (2007), necessariamente contém a Avaliação do desempenho estratégico, representada pela mensuração da performance de suas propriedades operacionais. Negligenciar isso pode significar uma falha no sucesso do empreendimento em estudo (DVIR et al., 1998).

O termo Avaliação de Desempenho pode ser visto sob diferentes pontos de vista. Assim, pode ser considerado um processo estruturado para coletar, descrever e representar dados, a fim de gerar informações sobre múltiplas dimensões de desempenho para diferentes usuários (BITITCl; CARRIE; MCDEVITT, 1997; NEELY; ADAMS; KENNERLEY, 2002; SHENHAR et al., 2002), que contempla aspectos financeiros e não financeiros e tem por objetivo oferecer informações para o aprendizado (KAPLAN; NORTON, 1997). Também pode ser considerado um processo estruturado que permite identificar, organizar, mensurar, integrar e gerenciar os elementos (critérios) segundo a percepção do decisor em um contexto único (ENSSLIN et al., 2010; LACERDA; ENSSLIN; ENSSLIN, 2011).

Nesse contexto, considera-se importante identificar os fundamentos da Avaliação de Desempenho. Assim, emerge a pergunta de pesquisa que orienta esta investigação: "Quais são os critérios que devem ser considerados em um processo estruturado que pretende identificar o referencial teórico com validade científica e promover revisão sistêmica que permita identificar potencialidades e oportunidades dos modelos de Avaliação de Desempenho?"

Para responder à pergunta de pesquisa, o objetivo geral deste trabalho é assim formulado: buscar referencial teórico com validade científica e avaliar o referencial selecionado para identificar as potencialidades e oportunidades dos modelos de Avaliação de Desempenho. Para atingir o objetivo geral, os seguintes objetivos específicos deverão ser perseguidos:

(i) identificar um portfólio de artigos internacionais com reconhecimento científico que aborde o tema Avaliação de Desempenho; 
(ii) promover análise sistêmica dos artigos selecionados. Para dar conta do objetivo estabelecido, selecionaram-se como instrumento de intervenção o processo estruturado de seleção de artigos e o processo de revisão sistêmica, ambos desenvolvidos pelo LabMCDA/UFSC, conforme Rosa, Ensslin e Ensslin (2009).

Após esta seção de caráter introdutório, a seção 2 apresenta 0 enquadramento metodológico da pesquisa e o procedimento para revisão da literatura; a seção 3 identifica as potencialidades e oportunidades do portfólio de artigos; e, por fim, a seção 4 apresenta as reflexões finais dos autores.

\section{METODOLOGIA DA PESQUISA}

Nesta seção, descreve-se a metodologia da pesquisa em termos do enquadramento metodológico e do processo para revisão de literatura.

\subsection{Enquadramento metodológico}

O enquadramento metodológico consiste na descrição das características da pesquisa segundo as bases científicas e na explicação do conjunto de procedimentos, ferramentas e abordagens utilizadas para dar conta da investigação proposta (TASCA et al., 2010).

Nesta pesquisa, o enquadramento metodológico é explicado pelos seguintes aspectos: natureza do objetivo; natureza do artigo; coleta de dados; abordagem do problema; procedimentos técnicos; resultados; e instrumento de pesquisa, conforme Quadro 1. 
Quadro 1 - Enquadramento metodológico

\begin{tabular}{|c|c|c|}
\hline Aspectos & Referência & Objeto ou Objetivo \\
\hline $\begin{array}{l}\text { Natureza do } \\
\text { objetivo }\end{array}$ & $\begin{array}{l}\text { Descritiva } \\
\text { (RICHARDSO } \\
\text { N et al., 2008) }\end{array}$ & $\begin{array}{l}\text { Identificar informações sobre Avaliação de Desempenho, } \\
\text { discutidas cientificamente nos artigos publicados em } \\
\text { periódicos internacionais. }\end{array}$ \\
\hline $\begin{array}{l}\text { Natureza do } \\
\text { artigo }\end{array}$ & $\begin{array}{l}\text { Teórico- } \\
\text { ilustrativa } \\
\text { (ALAVl; } \\
\text { CARLSON, } \\
\text { 1992) }\end{array}$ & $\begin{array}{l}\text { Compreender as pesquisas e buscar identificar suas } \\
\text { potencialidades e oportunidades. }\end{array}$ \\
\hline $\begin{array}{l}\text { Coleta de } \\
\text { dados }\end{array}$ & $\begin{array}{l}\text { Dados } \\
\text { secundários } \\
\text { (RICHARDSO } \\
\text { N et al., 2008) }\end{array}$ & $\begin{array}{l}\text { Utilizar artigos científicos internacionais publicados nas } \\
\text { bases de dados Oxford, Jstor, Science Direct, Scopus, } \\
\text { Compendex, EconLit, SAGE, Springer, Wilson, Blackwell, } \\
\text { ISI, Web of Science, Gale, Emerald, Cambridge e Science } \\
\text { Direct. }\end{array}$ \\
\hline \multirow[t]{2}{*}{$\begin{array}{l}\text { Abordagem } \\
\text { do problema }\end{array}$} & $\begin{array}{ll}\text { Qualitativa } & \\
\text { (SELLTIZ et } \\
\text { al., 1987) }\end{array}$ & $\begin{array}{l}\text { Identificar como a comunidade científica percebe a } \\
\text { Avaliação de Desempenho, a fim de identificar } \\
\text { oportunidades para promover/sugerir aperfeiçoamentos nos } \\
\text { processos de Avaliação de Desempenho. }\end{array}$ \\
\hline & $\begin{array}{l}\text { Quantitativa } \\
\text { (SELLTIZ et } \\
\text { al., 1987) }\end{array}$ & $\begin{array}{l}\text { Identificar o perfil dos artigos selecionados em termos da } \\
\text { quantidade de autores, periódicos, citações e períodos de } \\
\text { destaque. }\end{array}$ \\
\hline $\begin{array}{l}\text { Procedimento } \\
\text { s técnicos }\end{array}$ & $\begin{array}{l}\text { Bibliográfica } \\
\text { (BRUYNE; } \\
\text { HERMAN; } \\
\text { SCHOUTHEE } \\
\text { TE, 1991) }\end{array}$ & $\begin{array}{l}\text { Levantar dados estabelecidos na atual pesquisa para } \\
\text { identificar nos artigos selecionados qual é o tratamento dado } \\
\text { ao tema Avaliação de Desempenho. }\end{array}$ \\
\hline Resultados & $\begin{array}{l}\text { Aplicada } \\
\text { (RICHARDSO } \\
\text { N et al., 2008) }\end{array}$ & $\begin{array}{l}\text { Gerar conhecimentos sobre Avaliação de Desempenho por } \\
\text { meio de um arcabouço teórico sobre o assunto. }\end{array}$ \\
\hline $\begin{array}{l}\text { Instrumento } \\
\text { de pesquisa }\end{array}$ & $\begin{array}{l}\text { ProKnow-C } \\
\text { (TASCA et al., } \\
\text { 2010): } \\
\text { Bibliometria } \\
\text { (VANTI, 2002) } \\
\text { e } \\
\text { Revisão } \\
\text { sistêmica } \\
\text { (ENSSLIN, ET } \\
\text { AL 2010) }\end{array}$ & $\begin{array}{l}\text { Bibliometria - Traçar o perfil quantitativo dos artigos } \\
\text { selecionados e de suas referências, quanto aos aspectos: } \\
\text { análise por artigo; análise temporal; análise por periódico; } \\
\text { análise por autor; e relevância científica. } \\
\text { Revisão sistêmica - Identificar os seguintes aspectos: o } \\
\text { conceito (entendimento, interpretação) de Avaliação de } \\
\text { Desempenho; como é feita a identificação dos aspectos } \\
\text { relevantes nas abordagens analisadas; como é feita a } \\
\text { mensuração desses aspectos; como é feita a integração das } \\
\text { escalas; como é feito o diagnóstico; e como é feito o } \\
\text { aperfeiçoamento da situação atual. }\end{array}$ \\
\hline
\end{tabular}

Fonte: Elaborado pelos autores (2012).

\subsection{Procedimento para revisão da literatura}

O procedimento para revisão da literatura utilizado foi o processo ProKnow-

C (TASCA et al., 2010), que é realizado em três fases:

a) Seleção das bases de dados - Explicitação do Portal Mestre e seleção dos bancos de dados que serão utilizados (Fase 1 da Tabela 1).

Revista Produção Online, Florianópolis, SC, v.13, n. 2, p. 390-416, abr./jun. 2013. 
b) Seleção dos artigos para o referencial teórico - Definição das palavraschave para a realização da pesquisa; análise das publicações, tendo em vista a seleção de artigos alinhados com o contexto da pesquisa; seleção dos artigos com maior reconhecimento científico (pesquisa sobre citações dos artigos); exclusão pelos resumos; reexame dos artigos candidatos à exclusão pelo critério do número de citações; exclusão dos artigos cujo acesso gratuito ao texto completo não é ofertado pela CAPES; e relação dos artigos selecionados para a análise bibliográfica (Fase 2 da Tabela 1).

c) Análise bibliométrica dos artigos selecionados - A etapa anterior disponibiliza um portfólio bibliográfico de artigos que são, então, analisados quanto ao número de citações dos artigos, número de citações dos autores, periódicos dos artigos do portfólio bibliográfico, e palavras-chave do mesmo portfólio.

Tabela 1 - Dados quantitativos do processo de seleção dos artigos

\begin{tabular}{|c|c|c|c|}
\hline Fase & Etapa & Objetivo & $\begin{array}{l}\text { Resultado } \\
\text { quantitativo } \\
\text { alcançado }\end{array}$ \\
\hline 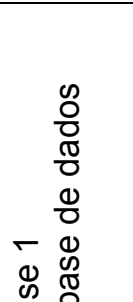 & A & $\begin{array}{l}\text { Identificar os temas (áreas de conhecimentos) vinculados à } \\
\text { pesquisa, e, em seguida, selecionar as bases de dados que } \\
\text { contemplem pelo menos um tema. Para tanto, consideraram-se as } \\
\text { áreas do conhecimento (segundo critérios da CAPES) vinculadas } \\
\text { ao tema de pesquisa: Multidisciplinar, Ciências Exatas e da Terra, } \\
\text { Ciências Sociais Aplicadas, Engenharias, Administração, } \\
\text { Contabilidade, Engenharia de Produção e Economia. }\end{array}$ & 110 \\
\hline 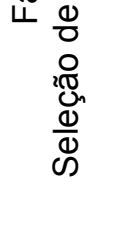 & B & $\begin{array}{l}\text { A seleção das bases de dados iniciou-se pela busca no site de } \\
\text { periódicos } \\
\text { (http://www.periodicos.capes.gov.br/portugues/index.jsp), } \\
\text { considerando bases de dados de textos completos e resumos que } \\
\text { contemplassem pelo menos uma das áreas de conhecimento } \\
\text { vinculadas ao tema, conforme critérios definidos na Fase } 1 .\end{array}$ & 16 \\
\hline$\frac{\pi}{\pi}$ & $\begin{array}{l}\text { A } \\
B\end{array}$ & $\begin{array}{l}\text { Definição das palavras-chave para realização da pesquisa. } \\
\text { Análise das publicações, tendo em vista a seleção de artigos } \\
\text { alinhados com o contexto da pesquisa. }\end{array}$ & $\begin{array}{c}8.003 \\
413\end{array}$ \\
\hline 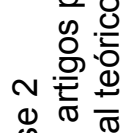 & C & $\begin{array}{l}\text { Seleção dos artigos com maior reconhecimento científico. Tal } \\
\text { reconhecimento científico foi mensurado pelo número de citações } \\
\text { dos artigos no Google Acadêmico (http://scholar.google.com.br). }\end{array}$ & 128 \\
\hline & D & Exclusão pelos resumos. & 15 \\
\hline 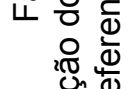 & $\mathrm{E}$ & $\begin{array}{l}\text { Reexame dos artigos candidatos à exclusão pelo critério do } \\
\text { número de citações. }\end{array}$ & 35 \\
\hline$\left.\frac{\omega}{\infty}\right)^{\Phi}$ & $\mathrm{F}$ & $\begin{array}{l}\text { Exclusão dos artigos cujo acesso gratuito ao texto completo não } \\
\text { era ofertado pela CAPES. }\end{array}$ & 26 \\
\hline & G & Apresentação da lista de artigos selecionados. & 26 \\
\hline
\end{tabular}

Fonte: Dados da pesquisa.

Revista Produção Online, Florianópolis, SC, v.13, n. 2, p. 390-416, abr./jun. 2013. 
A Tabela 1 apresenta a lógica do processo de seleção de artigos e os dados quantitativos obtidos em cada etapa do processo. A seguir, são descritas essa etapas.

\subsubsection{Seleção das bases de dados}

A primeira fase é composta de duas etapas, que, em conjunto e de forma sequencial, resultaram na seleção de 18 bases de dados. Segundo os critérios de seleção apresentados na Tabela 1, as bases de dados LISA e SocINDEX já estavam contidas na base de dados Wilson. Dessa forma, permaneceram 16 bases de dados para a busca dos artigos, quais sejam: Oxford, Jstor, Science Direct, Scopus, Compendex, EconLit, SAGE, Springer, Wilson, Blackwell, ISI, Web of Science, Gale, Emerald, Cambridge e Science Direct. As bases de dados com maior representatividade, no que se refere ao total de artigos selecionados, são: SAGE $(20,47 \%)$, Oxford $(16,71 \%)$ e Springer $(15,11 \%)$.

\subsubsection{Seleção dos artigos para compor o referencial teórico}

A segunda etapa, por sua vez, é composta de sete fases, conforme Tabela 1. Em conjunto e de forma sequencial, essa etapa resultou na seleção de 26 artigos, selecionados para compor o portfólio de artigos para o referencial teórico, que são analisados na revisão sistêmica apresentada na próxima seção desta pesquisa. A seguir, são apresentados os detalhes de cada etapa.

a) Definição das palavras-chave para a realização da pesquisa

Para a realização da pesquisa nas bases de dados selecionadas, foram definidas as seguintes palavras-chave: performance AND evaluation AND organization; performance AND assessment AND organization; performance AND measurement AND organization; performance AND appraisal AND organization; evaluation AND assessment AND organization; evaluation AND measurement AND organization; evaluation AND appraisal AND organization; assessment AND measurement AND organization; assessment AND appraisal AND organization; 
measurement AND appraisal AND organization. Nessa etapa, foram selecionados 8.003 artigos.

b) Análise das publicações, tendo em vista a seleção de artigos alinhados com o contexto da pesquisa

Esta etapa foi constituída das seguintes ações: importação das publicações para um software de gerenciamento bibliográfico, exclusão das publicações duplicadas, e exclusão dos artigos não alinhados com a área de interesse da pesquisa - análise do título. Após leitura de alguns títulos, foram identificadas algumas palavras-chave de exclusão: HIV, clinical, toxicologycol, biological, cardiacal, chemical, hospital, violence, children, patients, therapy, psychological, esquizofrenia, racism, veterinary, religio, photovoltaics, biotechnology, respiratory, ontology, nutritional, radiation, criminal, atmospheric, femininity, herbal, sexual, neuro, infants, cardio, cancer e police. Portanto, nessa análise de leitura dos títulos para verificação e exclusão dos artigos sem aderência à pesquisa, foram excluídas 5.126 publicações, restando 413.

c) Seleção dos artigos com maior reconhecimento científico

Tal reconhecimento científico foi mensurado pelo número de citações dos artigos no Google Acadêmico (http://scholar.google.com.br). Buscando selecionar os artigos com maior reconhecimento científico, foi realizada a pesquisa do número de citações de cada publicação remanescente. A pesquisa das citações foi realizada no Google Acadêmico (http://scholar.google.com.br) no dia 30 de março de 2009, e o resultado apurado é apresentado na Tabela 2. 
Tabela 2 - Número de citações por artigo

\begin{tabular}{lc}
\hline \multicolumn{1}{c}{ Artigo } & $\begin{array}{c}\text { N. de } \\
\text { citações }\end{array}$ \\
\hline ARON, J. Growth and Institutions: A Review of the Evidence. The World Bank Research & \\
Observer, v. 15, n. 1, p. 99-135, 2000. & 415 \\
JOINSON, A. N. Self-disclosure in computer-mediated communication: The role of self- & \\
awareness and visual anonymity. European Journal of Social Psychology, v. 31, n. 2, & \\
p. 177-192, 2001. & 294 \\
LEE, C.; LEE, K.; PENNINGS, J. M. Internal capabilities, external networks, and & \\
performance: a study on technology-based ventures. Strategic Management Journal, v. & \\
22, n. 6-7, p. 615-640, 2001. & 466 \\
MCEVILY, S. K.; CHAKRAVARTHY, B. The persistence of knowledge-based advantage: & \\
an empirical test for product performance and technological knowledge. Strategic & \\
Management Journal, v. 23, n. 4, p. 285-305, 2002. & 281 \\
YLI-RENKO, Helena; AUTIO, Erkko; SAPIENZA, H. J. Social capital, knowledge & \\
acquisition, and knowledge exploitation in young technology-based firms. Strategic & \\
Management Journal, v. 22, n. 6-7, p. 587-613, 2001. & 485 \\
\hline
\end{tabular}

Fonte: Elaborado pelos autores (2012).

Esse mesmo procedimento foi feito para todos os 413 artigos selecionados para essa fase do processo de seleção. Após este levantamento, foram considerados candidatos a integrar a base final os artigos que tiveram 15 ou mais citações. Dos 413 artigos remanescentes, 128 artigos foram selecionados como candidatos a integrar a base final.

\section{d) Exclusão pelos resumos}

Nesta etapa, foi feita a leitura do resumo dos 128 artigos candidatos à inclusão, sendo excluídos aqueles que não estavam alinhados com o tema Avaliação de Desempenho, segundo a percepção do pesquisador. Assim, foram excluídos 113 artigos, permanecendo 15 para a próxima etapa.

e) Reexame dos artigos candidatos à exclusão pelo critério do número de citações

Foi realizada a leitura dos títulos e resumos dos 285 artigos não selecionados na etapa descrita no item c, com o propósito de identificar aqueles que poderiam apresentar uma contribuição específica, ao estarem obrigatoriamente alinhados com a pesquisa e atenderem, pelo menos, a um dos seguintes critérios: atualidade (artigo publicado nos últimos dois anos, por não ter tido oportunidade de ser referenciado) e relevância do autor. Dos 285 artigos não selecionados, 20 artigos atenderam aos critérios definidos e foram incorporados 
como candidatos à base final. Assim, ela passou a contar com 35 artigos candidatos.

f) Exclusão dos artigos cujo acesso gratuito ao texto completo não era ofertado pela CAPES

Dos 35 artigos, somente permaneceram na base final aqueles em que 0 acesso ao texto completo era disponibilizado gratuitamente pela CAPES. Esse filtro adicional excluiu 09, e o portfólio de artigos ficou consolidado em 26.

g) Relação dos artigos selecionados para a análise sistêmica

O conjunto de etapas anteriores proporcionou a composição de um portfólio de 26 artigos, que, de forma fundamentada, passam a compor a amostra do referencial teórico desta pesquisa, conforme Quadro 2.

Quadro 2 - Relação dos artigos selecionados para a análise sistêmica (continua) ABERNETHY, M. A. et al. A multi-method approach to building causal performance maps from expert knowledge. Management Accounting Research, v. 16, n. 2, 135-155, 2005.

ALIGULIYEV, R. M. Performance evaluation of density-based clustering methods. Information Sciences, v. 179, n. 20, p. 3583-3602, 2009.

AZOFRA, V.; PRIETO B.; SANTIDRIÁN, A. The usefulness of a performance measurement system in the daily life of an organisation: a note on a case study. The British Accounting Review, v. 35, n. 4, p. 367-384, 2003.

BALASUBRAMANIAN, S; GUPTA, M. Structural metrics for goal based business process design and evaluation. Business Process Management Journal, v. 11, n. 6, p. 680-694, 2005.

BARBER, N.; SCARCELLI, J. M. Enhancing the assessment of tangible service quality through the creation of a cleanliness measurement scale. Managing Service Quality, v. 20, n. 1, p. 70-88, 2010.

BUYTENDIJK, F.; HATCH, T.; MICHELI, P.. Scenario-based strategy maps. Business Horizons, v. 53, n. 4, p. 335-347, 2010.

CHEN, Mu-Yen; HUANG, Mu-Jung; CHENG, Yu-Chen. Measuring knowledge management performance using a competitive perspective: An empirical study. Expert Systems with Applications, v. 36, n. 4, p. 8449-8459, 2009.

CHIN, Kwai-Sang; LO, Kwong-Chi; LEUNG, Jendy P.F. Development of user-satisfaction-based knowledge management performance measurement system with evidential reasoning approach.

Expert Systems with Applications, v. 37, n. 1, p. 366-382, 2010. 
Quadro 2 - Relação dos artigos selecionados para a análise sistêmica (conclusão)

DEW, N. et al. Affordable loss: behavioral economic aspects of the plunge decision. Strategic

Entrepreneurship Journal, v. 3, n. 2, p. 105-126, 2009.

DEY, P. K. Integrated project evaluation and selection using multiple-attribute decision-making technique. International Journal of Production Economics, v. 103, n. 1, p. 90-103, 2006.

FOSS, N. J. Bounded rationality and tacit knowledge in the organizational capabilities approach: an assessment and a re-evaluation. Industrial and Corporate Change, v. 12, n. 2, p. 185-201, 2003.

GIBBONS, R. Team theory, garbage cans and real organizations: some history and prospects of economic research on decision-making in organizations. Industrial and Corporate Change, v. 12, n. 4, p. 753-787, 2003.

HSU, Yueh-Ling; LI, Wen-Chin; CHEN, Kuang-Wei. Structuring critical success factors of airline safety management system using a hybrid model. Transportation Research Part E: Logistics and Transportation Review, v. 46, n. 2, p. 222-235, 2009.

$\mathrm{HU}$, Hsiu-Yuan et al. Using BPNN and DEMATEL to modify importance-performance analysis model - A study of the computer industry. Expert Systems with Applications, v. 36, n. 6, p. 99699979, 2009.

JANSSEN, R. et al. Decision support for integrated wetland management. Environmental Modelling \& Software, v. 20, n. 2, p. 215-229, 2005.

KETOKIVI, M. A.; SCHROEDER, R. G. Perceptual measures of performance: fact or fiction? Journal of Operations Management, v. 22, n. 3, p. 247-264, 2004.

LESKINEN, P. Measurement scales and scale independence in the analytic hierarchy process.

Journal of Multi-Criteria Decision Analysis, v. 9, n. 4, p. 163-174, 2000.

LI, Y.; LIAO, X. Decision support for risk analysis on dynamic alliance. Decision Support Systems, v. 42, n. 4, p. 2043-2059, 2007.

MITCHELL, R.; BOYLE, B. Knowledge creation measurement methods. Journal of Knowledge

Management, v. 14, n. 1, p. 67-82, 2010.

MOHAMADABADI, H. S.; TICHKOWSKY, G.; KUMAR, A. Development of a multi-criteria assessment model for ranking of renewable and non-renewable transportation fuel vehicles.

Energy, v. 34, n. 1, p. 112-125, 2009.

OLSMATS, C.; DOMINIC, C. Packaging scorecard - a packaging performance evaluation method.

Packaging Technology and Science, v. 16, n. 1, p. 9-14, 2003.

ÖNÜT, S.; KARA, S. S.; IŞIK, E. Long term supplier selection using a combined fuzzy MCDM approach: A case study for a telecommunication company. Expert Systems with Applications, v. 36, n. 2/part 2, p. 3887-3895, 2009.

ROUWETTE, E. A. J. A.; VENNIX, J. A. M.; MULLEKOM, T. v. Group model building effectiveness: a review of assessment studies. System Dynamics Review, v. 18, n. 1, p. 5-45, 2002.

SELDEN, S. C.; SOWA, J. E. Testing a Multi-Dimensional Model of Organizational Performance: Prospects and Problems. Journal of Public Administration Research and Theory. v. 14, n. 3, p. 395-416, 2004.

WORREN, N.; MOORE, K.; CARDONA, P. Modularity, strategic flexibility, and firm performance: a study of the home appliance industry. Strategic Management Journal, v. 23, n. 12, p. 1123-1140, 2002.

WOUTERS, M.; WILDEROM, C. Developing performance-measurement systems as enabling formalization: A longitudinal field study of a logistics department. Accounting, Organizations and Society, v. 33, n. 4-5, p. 488-516, 2008.

Fonte: Elaborado pelos autores (2012). 
Transcorridas as duas primeiras fases do processo para revisão da literatura, executa-se a terceira fase, denominada análise bibliométrica, sendo feita para os 26 artigos selecionados.

\subsubsection{Análise bibliométrica dos artigos selecionados}

A análise bibliométrica é entendida como forma de medição voltada para avaliar a ciência e os fluxos da informação (VANTI, 2002). A presente pesquisa faz uso do processo ProKnow-C (TASCA et al., 2010), que define a análise bibliométrica como o processo de evidenciação dos dados estatísticos de um conjunto definido de artigos (Portfólio Bibliográfico) para a gestão da informação e do conhecimento científico de um dado assunto, realizado por meio da contagem de documentos.

A análise bibliométrica é composta de três etapas: (i) análise dos artigos do portfólio; (ii) análise das referências dos artigos do portfólio; e (iii) análise da relevância dos artigos e suas referências.

A análise bibliométrica dos 26 artigos selecionados revelou que WORREN, MOORE e CARDONA (2002) são os autores cujo artigo é o mais citado; 2009 é o ano com maior concentração de artigos; Expert Systems With Applications é o periódico com maior participação no portfólio bibliográfico selecionado. Todos os autores participaram com apenas 01 artigo da amostra.

Já a análise bibliométrica das 1.182 referências dos 26 artigos selecionados revelou que os anos com maior concentração de artigos são 1996, 2000 e 2002; Strategic Management Journal é o periódico de maior destaque, aparecendo 30 vezes; o autor mais citado é Dey (13 citações). O Centre for Business Performance, da Universidade de Cranfield, é uma referência no assunto, com uma produção muito acentuada de 1999 a 2006.

Por fim, pela observação dos artigos constantes na base final (26 artigos) e de suas 1.182 referências bibliográficas, foi possível perceber que: (i) Cardona, Moore, Woreen e Rouwette são autores que se destacam pelo elevado número de citações no Google Acadêmico (http://scholar.google.com.br); (ii) Janssen e Foss são os autores que se destacam pelo elevado número de citações nas referências utilizadas nos artigos da amostra; (iii) Ketokivi e Schroeder aparecem nas duas 
dimensões consideradas, sendo, portanto, o autor de destaque na amostra de artigos selecionados.

\section{REVISÃO SISTÊMICA}

A revisão sistêmica é entendida como um processo científico utilizado para, com base em uma amostra estabelecida de pesquisas e eixos de avaliação definidos (olhar, foco, problema): (i) identificar e sintetizar os conceitos utilizados e relacionados com a Avaliação de Desempenho e o assunto da pesquisa (eixos da pesquisa); (ii) identificar os aspectos relevantes; (iii) mensurar os aspectos relevantes; (iv) integrá-los; (v) identificar o diagnóstico atual; e (vi) oferecer informações para o aperfeiçoamento da situação (ENSSLIN; ENSSLIN, 2010).

Embasada nesse conceito, a revisão sistêmica é composta pelas seis fases citadas, sendo utilizada para identificar as potencialidades de oportunidades do portfólio de 26 artigos.

\subsubsection{Conceito (entendimento, interpretação) de Avaliação de Desempenho}

A análise do conceito de Avaliação de Desempenho é feita sob duas perspectivas: identificação e explicitação do conceito utilizado no artigo, e entendimento e interpretação do conceito frente aos paradigmas de Apoio à Decisão.

\subsubsection{Identificação e explicitação do conceito (entendimento, interpretação) de Avaliação de Desempenho utilizado no artigo}

A partir do estudo feito nos artigos, foi possível verificar que a Avaliação de Desempenho é um processo para analisar problemas complexos que envolve múltiplos critérios de decisão e fatores subjetivos e objetivos a serem considerados no processo decisório e tem o intuito de fornecer aos gerentes uma base racional para tomada de decisões Mohamadabadi, Tichkowsky e Kumar (2009).

Considera-se ainda que o contexto da Avaliação de Desempenho seja singular (ABERNETHY et al., 2005), sendo utilizado para analisar processos 
(BALASUBRAMANIAN; GUPTA, 2005), identificar pontos fortes e fracos do contexto e melhorar a aprendizagem contínua (CHIN; LO; LEUNG, 2010).

Avaliação de Desempenho também é utilizada para mensurar aspectos financeiros e não financeiros com base no mapa estratégico da empresa (AZOFRA; PRIETO; SANTIDRIÁN, 2003) e para construir cenários futuros baseados em mapas estratégicos, considerando as preocupações dos stakeholders (BUYTENDIJK; HATCH; MICHELI, 2010).

Serve ainda para analisar as relações entre as variáveis e estabelecer como os gestores decidem na prática (WORREN; MOORE; CARDONA, 2002), se estão apoiados em critérios e subcritérios de análise identificados por especialistas (LI; LIAO, 2007).

No processo de Avaliação de Desempenho, é importante ter meios para importar o conhecimento tático de especialistas para gerar o conhecimento organizacional (ABERNETHY et al., 2005). Esse processo de Avaliação de Desempenho é construído a partir da percepção dos analistas com a finalidade de sugerir ou prescrever como os gestores devem tomar decisões e possibilitar a geração de alternativas e comparação entre elas para solucionar o problema avaliado (HSU; LI; CHEN, 2009).

A Avaliação de Desempenho requer um processo estruturado (ÖNÜT; KARA; IŞIK, 2009; PARK; KWANGTAE, 2009; KUMAR, 2008), ou um conjunto de critérios estabelecidos pelos especialistas (BARBER; SCARCELLI, 2010; CHEN; HUANG; CHENG, 2009), ou, ainda, um conjunto de critérios preestabelecidos na literatura (ROUWETTE; VENNIX, 2002), para auxiliar na tomada de decisão a respeito da escolha de alternativas.

Alguns dos modelos resultam também em um conjunto de alternativas reais que podem se alterar durante sua construção e servem também para gerar novas alternativas (HU et al., 2009; JANSSENA et al., 2005; SELDEN; SOWA, 2004; WOUTERS; WILDERON, 2008; CARL; CHRIS, 2003).

\subsubsection{Análise do conceito (entendimento, interpretação) de Avaliação de Desempenho frente aos paradigmas de Apoio à Decisão}


Segundo Ensslin e Ensslin (2009), avaliar o desempenho serve para construir um conhecimento do contexto que permita tomar decisões com compreensão de suas consequências naquilo que o decisor julga ser importante. Assim, um modelo de avaliação está baseado nos seis paradigmas: (i) singularidade; (ii) conhecimentos limitados; (iii) entidade social; (iv) recursividade da aprendizagem participativa; (v) princípios da mensuração; e (vi) legitimidade e validação. Com base nesses paradigmas, é feita a análise dos 26 artigos selecionados, identificando-se os pontos fortes e oportunidades, conforme Quadro 3.

Quadro 3 - Análise do artigos frente aos paradigmas do construtivismo (continua)

\begin{tabular}{|c|c|}
\hline $\begin{array}{l}\text { Paradigma do Apoio } \\
\text { à Decisão }\end{array}$ & Pontos fortes e oportunidades \\
\hline $\begin{array}{l}\text { P1= Singularidade, } \\
\text { identidade: os valores } \\
\text { e preferências do } \\
\text { decisor }\end{array}$ & $\begin{array}{l}\text { Chen, Huang e Cheng (2009) reconhecem que o contexto analisado } \\
\text { é singular, portanto, os modelos são construídos para auxiliar os } \\
\text { decisores a gerar conhecimento (aprender) sobre seu problema. Os } \\
\text { demais artigos analisados utilizam critérios preestabelecidos para } \\
\text { avaliar desempenho. Assim, os modelos não são singulares e não } \\
\text { expressam os valores daquele que toma decisões. }\end{array}$ \\
\hline $\begin{array}{l}\text { P2 = Conhecimentos } \\
\text { limitados: a } \\
\text { necessidade do } \\
\text { decisor em melhorar } \\
\text { seu entendimento } \\
\text { sobre as } \\
\text { consequências da } \\
\text { decisão. }\end{array}$ & $\begin{array}{l}\text { Azofra, Prieto e Alicia (2003) e Buytendijk, Hatch e Pietro (2010) } \\
\text { reconhecem a importância de gerar conhecimento no decisor nas } \\
\text { fases de (i) construção dos indicadores e (ii) construção dos } \\
\text { cenários, respectivamente. Os demais artigos analisados não geram } \\
\text { conhecimento no decisor, pois parte de critérios e subcritérios } \\
\text { preestabelecidos (literatura ou especialistas) descrevem os critérios } \\
\text { e subcritérios a partir do contexto apresentado pelos envolvidos no } \\
\text { processo de tomada de decisão. }\end{array}$ \\
\hline $\begin{array}{l}\text { P } 3 \text { = Entidade } \\
\text { Social: as } \\
\text { organizações estão } \\
\text { inseridas em um } \\
\text { sistema aberto e, } \\
\text { portanto, sofrem } \\
\text { influência do meio } \\
\text { externo, sendo } \\
\text { necessário conhecer } \\
\text { o contexto na qual } \\
\text { está inserido o } \\
\text { problema. }\end{array}$ & $\begin{array}{l}\text { Segundo Abernethy et al. (2005) e Azofra, Prieto e Alicia (2003), } \\
\text { existem diversos atores envolvidos em um processo decisório, e o } \\
\text { modelo é construído a partir da percepção dos gestores.Buytendijk, } \\
\text { Pietro e Hatch (2010), Carl e Chris (2003), Chen, Huang e Cheng } \\
\text { (2009), Chin, Lo e Leung (2010), Önüt, Kara e Işik (2009) } \\
\text { reconhecem que existem diversos grupos de interesse na decisão. } \\
\text { Assim, deve-se levar em conta os valores de tais grupos, porém o } \\
\text { facilitador infere e testa esses valores. Os valores/critérios são } \\
\text { identificados a partir de questionários, entrevistas e validação dos } \\
\text { critérios identificados na literatura pelos decisores e inferidos pelo } \\
\text { facilitador. Hu et al. (2009), Worren, Moore e Cardona (2002) e } \\
\text { Selden e Sowa (2004) reconhecem que existem uma série de grupos } \\
\text { e indivíduos envolvidos na decisão, portanto deve-se descrever suas } \\
\text { relações e seus papéis, sendo que o(s) decisor(es) não participa(m) } \\
\text { para expressar seus valores e interesses. Já Aliguliyev (2009), } \\
\text { Balasubramanian e Gupta (2005), Barber e Scarcelli (2010), } \\
\text { Rouwette e Vennix (2002), Gibbons (2003), Hsu, Li e Chen (2009), } \\
\text { Janssena et al. (2005), Park e Kwangtae (2009), Mohamadabadi, } \\
\text { Tihkowsky, Kumar (2008) supõem um decisor completamente } \\
\text { racional, que não necessite interagir com os decisores reais. Assim, } \\
\text { as soluções não estão necessariamente alinhadas com os objetivos } \\
\text { do decisor, pois este não participa para expressar seus valores e } \\
\text { interesses. Dessa forma, identificam-se os critérios através da } \\
\text { literatura. }\end{array}$ \\
\hline
\end{tabular}


Quadro 3 - Análise do artigos frente aos paradigmas do construtivismco (conclusão)

\begin{tabular}{|c|c|}
\hline $\begin{array}{l}\text { P } 4 \text { = Recursividade } \\
\text { da aprendizagem } \\
\text { participativa: a } \\
\text { dinâmica recursiva do } \\
\text { processo de } \\
\text { aprendizagem das } \\
\text { partes envolvidas. }\end{array}$ & $\begin{array}{l}\text { O modelo é construído. Aqui o decisor participa do processo de } \\
\text { construção do modelo de avaliação (BUYTENDIJK, PIETRO E } \\
\text { HATCH, 2010; Dey, 2006; WORREN, MOORE e CARDONA, 2002). } \\
\text { Para Azofra, Prieto e Santidrián (2003), Hu et al. (2009), Selden e } \\
\text { Sowa (2004) e Wouters e Wilderon (2008), os decisores não fazem } \\
\text { parte do processo, pois o importante é descrever o comportamento } \\
\text { deles na tomada de decisão. Já para Aliguliyev (2009), } \\
\text { Balasubramanian e Gupta (2005), Barber e Scarcelli (2010), } \\
\text { Rouwette e Vennix (2002), Gibbons (2003), Hsu, Li e Chen (2009), } \\
\text { Janssena et al. (2005), Li e Liao (2007), Park e Kwangtae (2009) e } \\
\text { Safaei Mohamadabdi, Tihkowsky, Kumar (2008), o analista estrutura } \\
\text { o modelo sem a participação dos decisores, pois assumem que o } \\
\text { decisor é racional. E, por fim, para Abernethy et al. (2005), Carl e } \\
\text { Chris (2003), Chen, Huang e Cheng (2009), Ching, Lo e Leung } \\
\text { (2010) e Önüt, Kara e Işik (2009), os decisores são ouvidos, pois os } \\
\text { valores desses devem ser identificados, e, após a identificação dos } \\
\text { valores do decisores, não é mais necessária a participação deles. }\end{array}$ \\
\hline $\begin{array}{l}\text { P } 5 \text { = Princípios da } \\
\text { mensuração: as } \\
\text { propriedades das } \\
\text { escalas ordinais de } \\
\text { intervalo e de razão. }\end{array}$ & $\begin{array}{l}\text { Pode-se constatar que alguns modelos estão focados apenas em } \\
\text { identificar critérios de avaliação (Abernethy et al. 2005; Buytendijk, } \\
\text { Pietro e Hatch, 2010; Rouwette e Vennix, 2002; e Hsu, Li e Chen, } \\
\text { 2009); e aqueles que permitem mensurar aquilo que se propõem a } \\
\text { avaliar utilizam-se de escalas tipo Likert (Azofra, Peitro e Alicia, } \\
\text { 2003; Carl e Chris, 2003; Chen, Huang e Cheng, 2009; Chin e Lo, e } \\
\text { Leng, 2010; Hu et al., 2009; Janssena et al., 2005; Li e Liao, 2007; } \\
\text { Worren, Moore e Cardona, 2002; Önüt, Kara e Işik, 2009; Park e } \\
\text { Kwangtae, 2009); e Safaei Mohamadabdi, Tihkowsky, Kumar, 2008). } \\
\text { Para Aliguliyev (2009); Balasubramanian e Gupta (2005); Barber e } \\
\text { Scarcelli (2010); Selden e Sowa (2004); e Wouters e Wilderon } \\
\text { (2008), a mensuração é feita por meio de atribuição de pesos ou } \\
\text { médias ponderadas. Contudo, para Krumar (2008), a mensuração é } \\
\text { feita por meio de tabulação de dados sobre os critérios quantificados } \\
\text { para auxiliar nas escolhas entre alternativas. }\end{array}$ \\
\hline $\begin{array}{l}\text { P } 6 \text { = Validação e } \\
\text { Legitimidade: o } \\
\text { reconhecimento } \\
\text { científico e o } \\
\text { reconhecimento } \\
\text { social gerado no } \\
\text { processo de } \\
\text { construção de } \\
\text { conhecimento. }\end{array}$ & $\begin{array}{l}\text { Todos os artigos possuem validação científica. Contudo, nem todos } \\
\text { os modelos reconhecem que haja participação do decisor na } \\
\text { construção do modelo, mas alguns autores reconhecem a } \\
\text { importância da participação do decisor, dessa forma, nesses artigos, } \\
\text { é demonstrada a participação parcial dos decisores. Assim, para } \\
\text { Buytendijk e Prietro (2010), a construção de mapas é feita a partir } \\
\text { dos objetivos estratégicos da organização e validados pelo } \\
\text { decisor.Para Carl e Chris (2003) os critérios preestabelecidos devem } \\
\text { ser validados pelo decisor, e, finalmente, para Chen, Huang e Cheng } \\
\text { (2009); Ching, Lo e Leung (2010); e Kumar (2008), os decisores são } \\
\text { ouvidos pelo facilitador nas etapas de identificação e mensuração } \\
\text { dos critérios de avaliação, e a partir daí o facilitador aufere para } \\
\text { encontrar as alternativas para o problema analisado. } \\
\text { Para os demais artigos analisados, os decisores não participam do } \\
\text { processo de construção do modelo, portanto não são modelagens } \\
\text { que permitem gerar conhecimento naquele que toma decisão. } \\
\text { (Abernethy et al., 2005; Aliguliyev, 2009; Azofra, Prieto e Santidrián, } \\
\text { 2003; Buytendijk, Pietro e Hatch, 2010; Barber e Scarcelli, 2010; } \\
\text { Rouwette e Vennix, 2002; Hsu, Li e Chen, 2009; Hu et al., 2009; } \\
\text { Janssena et al., 2005; Li e Liao, 2007. }\end{array}$ \\
\hline
\end{tabular}

Fonte: Elaborado pelos autores (2012).

A partir do Quadro 3, é possível constatar que a maioria dos autores se vale

de critérios preestabelecidos para identificar o que é importante avaliar, não Revista Produção Online, Florianópolis, SC, v.13, n. 2, p. 390-416, abr./jun. 2013. 
reconhecendo a necessidade de o decisor aprofundar seu entendimento sobre as consequências da decisão. Ou seja, o modelo é estruturado visando descrever o comportamento dos decisores na tomada de decisão, ou o especialista estrutura o modelo sem a participação dos decisores, pois ele assume um decisor racional, ou, ainda, os decisores são ouvidos pelo analista, visando obter seus valores e, após estes serem obtidos, não é mais necessária a participação dos decisores.

Foi possível perceber também que os autores dos artigos analisados reconhecem que os decisores sofrem influência do meio externo, sendo necessário conhecer o contexto no qual está inserido o problema. No entanto, não foi reconhecida, nos artigos analisados, a percepção do decisor para a construção de todas as etapas de determinado modelo de avaliação.

\subsection{Identificação dos aspectos relevantes}

A identificação dos aspectos relevantes ocorre por meio da identificação dos meios e da forma de participação do decisor no processo.

\subsubsection{Meios para identificação dos aspectos relevantes}

Os aspectos relevantes em um modelo de Avaliação de Desempenho, segundo Keeney (1992), podem ser: o objetivo, o critério, o atributo, o fator crítico de sucesso e ponto de vista fundamental.

Abernethy et al. (2005); Buytendijk, Hatch e Pietro (2010); Worren, Moore e Cardona (2002); Önüt, Kara e Işik (2009); Selden e Sowa (2004); Wouters e Wilderon (2008) identificam os critérios por meio de entrevistas e questionários. Já Aliguliyev (2009) identifica os critérios utilizando um software que organiza em clusters critérios com características semelhantes em documentos internos da empresa analisada. Já para Azofra, Prieto e Alicia (2003); Balasubramanian e Gupta (2005); Barber e Scarcelli (2010); Carl e Chris (2003); Chen, Huang, Cheng (2009); Rouwette e Vennix (2002); Hsu, Li e Chen (2010) e Hu et al. (2009), os critérios e subcritérios são identificados na literatura. Chin, Lo e Leung (2010); Dey (2006); Li e Liao (2007); Park e Kwangtae (2009) e Kumar (2008) baseiam-se no 
conhecimento de especialistas. E finalmente Carl e Chris (2003) identificam os critérios na estratégia da organização estudada.

Portanto, a etapa de identificação é fundamental para o processo de construção de modelos de avaliação, sendo observadas diferentes abordagens para obter um conjunto de elementos que irão compor o modelo. Assim sendo, pode-se valer do conhecimento empírico de especialistas, do conhecimento teórico obtido na literatura e do conhecimento tácito de decisores para a identificação desses elementos.

\subsubsection{Forma de participação do decisor no processo}

Resgatando o conceito de Avaliação de Desempenho, consideramos que o decisor deva participar de todo o processo de construção do modelo de Avaliação de Desempenho. Dessa forma, buscou-se identificar a partipação ou não dos decisores nesse processo.

Para Buytendijk, Pietro e Hatch (2010), a construção de mapas é feita a partir dos objetivos estratégicos da organização e validados pelo decisor, Carl e Chris (2003) demonstram que os critérios preestabelecidos devem ser validados pelo decisor; e, finalmente, para Chen, Huang e Cheng (2009), Ching, Lo e Leung (2010) e Kumar (2008), os decisores são ouvidos pelo facilitador nas etapas de identificação e mensuração dos critérios de avaliação, e, a partir daí, o facilitador aufere para encontrar as alternativas ao problema analisado.

Para os demais artigos analisados, os decisores não participam do processo de construção do modelo, portanto não são modelagens que permitem gerar conhecimento naquele que toma decisão: Abernethy et al. (2005); Aliguliyev (2009); Azofra, Prieto e Santidrián (2003); Buytendijk, Pietro e Hatch, 2010; Barber e Scarcelli (2010); Rouwette e Vennix (2002); Hsu, Li e Chen (2009); Hu et al. (2009); Janssena et al. (2005); Li e Liao (2007).

\subsection{Mensuração dos aspectos relevantes (construção e integração de escalas de mensuração)}


Segundo Ensslin e Ensslin, S. (2009), os indicadores de desempenho são uma parte da Avaliação de Desempenho e não podem ser dela dissociados. Os indicadores possuem as seguintes propriedades: são mensuráveis, operacionais, inteligíveis, permitem distinguir o desempenho melhor e pior, e respeitam as propriedades das escalas ordinais.

Com a finalidade de identificar como é feita a mensuração nos artigos analisados, descrevem-se as escalas utilizadas e seus mecanismos de integração.

É possível verificar também que algumas pesquisas estão focadas apenas em identificar critérios de avaliação (Abernethy et al., 2005; Buytendijk, Pietro e Hatch, 2010; Rouwette e Vennix, 2002; e Hsu, Li e Chen, 2009), e aqueles que permitem mensurar aquilo que se propõe a avaliar não contemplam a teoria da mensuração.

Assim, pode-se verificar que algumas pesquisas analisadas utilizam escalas tipo Likert (Azofra, Prieto e Santidrián, 2003; Carl e Chris, 2003; Chen, Huang e Cheng, 2009; Chin e Lo, e Leng, 2010; Hu et al., 2009; Janssena et al., 2005; Li e Liao, 2007; Worren, Moore e Cardona, 2002; Önüt, Kara e Işik, 2009; Park e Kwangtae, 2009; e Safaei Mohamadabdi, Tihkowsky, Kumar, 2008.

Verifica-se ainda que, para Aliguliyev (2009), Balasubramanian e Gupta (2005), Barber e Scarcelli (2010), Selden e Sowa (2004), e Wouters e Wilderon (2008), a mensuração é feita por meio de atribuição de pesos ou médias ponderadas.

Contudo, para Krumar (2008) a mensuração é feita por meio de tabulação de dados sobre os critérios quantificados para auxiliar na escolhas entre alternativas.

A integraçao das escalas de mensuração permite agregar os vários critérios identificados pelos decisores como importantes, e mensurados atravéz de escalas não ambíguas, homogêneas, ordinais e cardinais.

A análise permitiu identificar que a integração é feita por tabulação ou por ponderação (Azofra, Prieto e Santidrián, 2003; Carl e Chris, 2003; Chen, Huang e Cheng, 2009; Chin e Lo, e Leng, 2010; Hu et al., 2009; Janssena et al., 2005; Li e Liao, 2007; Worren, Moore e Cardona, 2002; Önüt, Kara e Işik, 2009; Park e Kwangtae, 2009); e Safaei Mohamadabdi, Tihkowsky, Kumar, 2008. 


\subsection{Diagnóstico atual e aperfeiçoamento da situação}

Segundo Ensslin et al. (2007), o maior potencial do uso de modelos de Avaliação de Desempenho está em sua capacidade de comunicar, dar transparência, fundamentar, alinhar, mas principalmente, de construir entendimento sobre aquilo que se está avaliando. Pesquisas em desenvolvimento no LabMCDA-UFSC mostram que o grau de sucesso das decisões é uma função do grau de entendimento que o decisor tem do contexto que gerencia, conforme Figura 2.

Figura 2 - O potencial de sucesso das decisões em função do grau de conhecimento do Decisor do Contexto

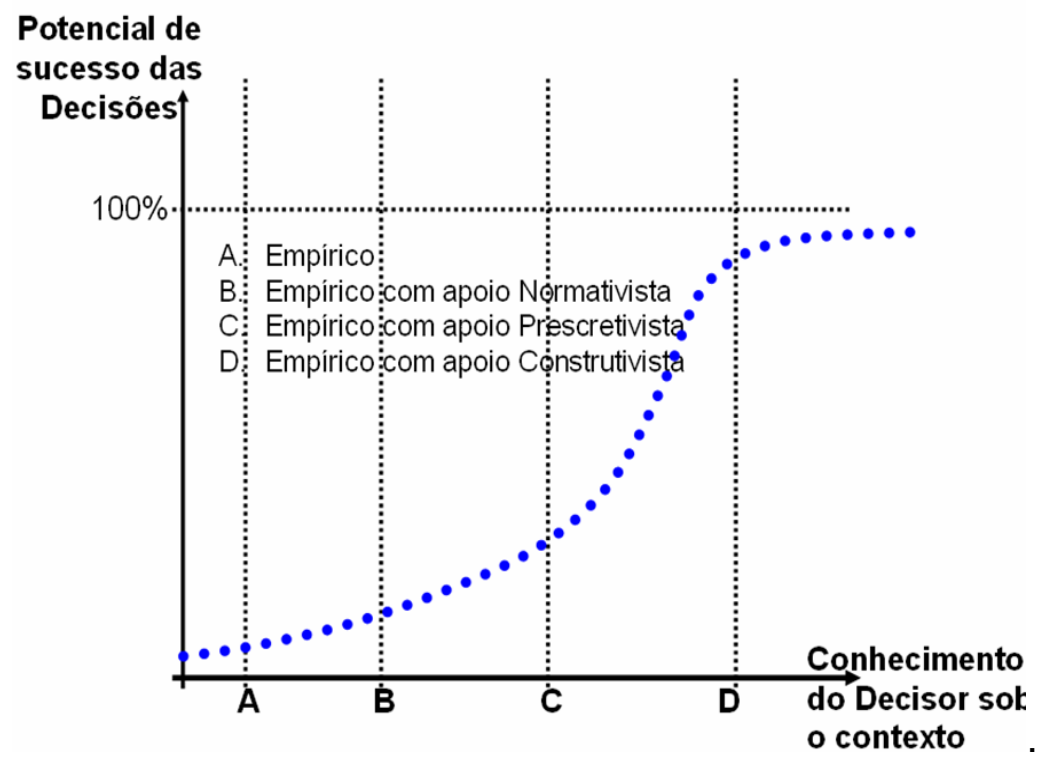

Fonte: Ensslin e Ensslin (2009).

Os artigos analisados apresentam modelos que se valem de tabulações e ponderações ou apresentação de conjuntos de alternativas para apresentar o diagnóstico do status quo. Assim, entende-se que os resultados representam normas de comportamento racional, ou seja, os resultados são verdades para o decisor racional. Além disso, os resultados são vistos como prescrições, que devem ser seguidas pelos decisores, para manter a sua coerência lógica.

A escolha do modelo a ser utilizado, assim como sua operacionalização, deve levar em conta suas consequências futuras em termos do alinhamento das consequências com os objetivos e valores almejados pela organização, bem como pelo grau de comprometimento das pessoas envolvidas (ENSSLIN et al., 2007). 


\section{CONCLUSÕES}

Visando identificar as potencialidades e oportunidades em investigações internacionais sobre Avaliação de Desempenho, esta pesquisa buscou responder à sua pergunta norteadora: "Quais os critérios que devem ser considerados em um processo estruturado que pretende identificar um referencial teórico com validade científica e uma revisão sistêmica que permita identificar potencialidades e oportunidades dos modelos de avalaição de desempenho?"

Nesse contexto, seu objetivo geral foi assim definido: buscar referencial teórico com validade científica para identificar potencialidades e oportunidades de modelos de Avaliação de Desempenho.

Buscou-se atender a esse objetivo por meio dos seus objetivos específicos: (i) identificar um portfólio de artigos internacionais com reconhecimento científicos que pesquisas o tema Avaliação de Desempenho; (ii) promover análise sistêmica dos artigos selecionados. Para dar conta do objetivo estabelecido, selecionou-se, como instrumento de intervenção, um processo estruturado de seleção de artigos e um processo de revisão sistêmica, ambos desenvolvidos pelo LabMCDA/UFSC.

Assim, para atender o primeiro objetivo específico, utilizou-se de um processo estruturado composto de três fases: (i) seleção das bases de dados; (ii) seleção de artigos para o referencial teórico; e (iii) análise bibliométrica dos artigos selecionados. Este processo, portanto, permitiu selecionar de forma fundamentada 26 artigos internacionais com validação científica, e, a partir dessa seleção, promoveu análise bibliométrica. Nessa análise bibliométrica, constatou-se a relevância da amostra de artigos (26) e de suas referências (1.182).

Dessa forma, para os 26 artigos identificou-se que: Modularity, strategic flexibility, and firm performance: a study of the home appliance industry. Strategic Management Journal, v. 23, n.12, p. 1123-1140 (WORREN, N; MOORE, K.; CARDONA, P., 2002) é o artigo mais citado; 2009 é o ano com maior concentração de artigos; Expert Systems With Applications é o periódico com maior participação; e todos os autores participaram da amostra com apenas 1 artigo.

Já nas 1.182 referências utilizadas pelos 26 artigos da amostra foi possível perceber que: (i) 1996, 2000 e 2002 são os anos com maior concentração de 
artigos, (ii) Strategic Management Journal é o periódico de maior destaque, aparecendo 30 vezes, e (iii) Dey, P. K. é o autor mais citado (13 citações).

Adicionalmente, verificou-se a correlação entre o reconhecimento científico dos autores e o reconhecimento científico dos artigos, buscando evidenciar os artigos de maior destaque. Dessa forma, foi possível perceber que: (i) Cardona, Moore e Woreen e Rouwette são autores que se destacam pelo elevado número de citações no google acadêmico (http://scholar.google.com.br); (ii) Janssena, R. e Foss, N. J., são autores que se destacam pelo elevado número de citações nas referências utilizadas nos artigos da amostra; (iii) Schroeder, R. G. é autor de destaque nas duas dimensões consideradas, sendo, portanto, o autor de destaque na amostra de artigos selecionados.

Após essa etapa de seleção de artigos e análise bibliométrica, buscou-se atender ao segundo objetivo específico desta pesquisa, promovendo análise sistêmica dos 26 artigos. Para tanto, foram considerados os seguintes aspectos de análise: 1. conceitos utilizados relacionados com a Avaliação de Desempenho e o assunto da pesquisa (eixos da pesquisa); 2. Identificação dos aspectos relevantes; 3. Mensuração dos aspectos relevantes; 4. Integração dos aspectos relevantes; 5. Diagnóstico atual; e 6. Aperfeiçoamento da situação.

Nessa análise dos 26 artigos, é possível verificar que:

a) Não há consenso quanto a definição sobre Avaliação de Desempenho;

b) A esmagadora maioria dos artigos constrói modelos a partir de critérios preestabelecidos pela literautura ou por especialistas;

c) Os modelos apresentados carecem de escalas de mensuração que permitam distinguir o desempenho melhor e pior e que respeitem as propriedades das escalas ordinais e cardinais;

d) Os modelos também carecem de integração de escalas que permitam identificar o grau de importância de cada critério de forma individual e seu impacto na avaliação global;

e) Os modelos buscam apresentar o diagnóstico do problema avaliado e carecem de processo de aperfeiçoamento do status quo.

Verificou-se, portanto que, nos artigos analisados, a construção de modelos de Avaliação de Desempenho requer o conhecimento de aspectos considerados Revista Produção Online, Florianópolis, SC, v.13, n. 2, p. 390-416, abr./jun. 2013. 
relevantes para um decisor ou especilista que está construindo o modelo para avaliar um contexto específico. No entanto, reconhecemos que a Avaliação de Desempenho é um processo que permite identificar, organizar, mensurar, integrar e gerenciar os elementos (critérios) segundo a percepção do(s) decisor(es) em um contexto único, considerando importante utilizar um instrumento para construir o entendimento de forma a permitir pró-ativamente conhecer as consequências das decisões e/ou alternativas, segundo a percepção dos gestores.

Compreende-se, ainda, que os decisores possuem conhecimentos limitados, havendo a necessidade de o decisor melhorar seu entendimento sobre as consequências da decisão; portanto, deve-se permitir que o decisor participe da construção de todas as etapas do modelo de Avaliação de Desempenho (identificação, organização, mensuração, integração e gerenciamento).

A partir desse pressuposto, compreende-se que a avalição de desempenho conduz a um futuro desejado e planejado e ao gerenciamento interno das informações. Entende-se que, para avaliar o desempenho organizacional, é fundamental ter a compreensão do contexto, representado por um modelo que evidencie os aspectos importantes estrategicamente e como esses aspectos se relacionam por meio da explicitação de suas atividades operacionais. Portanto, é imprescindível preocupar-se com a quantidade e qualidade das informações disponíveis, bem como com o ciclo de feedback dessas informações no processo de gestão.

\section{REFERÊNCIAS}

ABERNETHY, M. A. et al. A multi-method approach to building causal performance maps from expert knowledge. Management Accounting Research, v. 16, n. 2, p. 135-155, 2005.

ALIGULIYEV, R. M. Performance evaluation of density-based clustering methods. Information Sciences, v. 179, n. 20, p. 3583-3602, 2009.

ALAVI, M.; CARLSON, P. A Review of MIS Research and Disciplinary Development. Journal of Management Information Systems, v. 8, n. 4, p. 45-62, 1992.

ARON, J. Growth and Institutions: A Review of the Evidence. The World Bank Research Observer, v. 15, n. 1, p. 99-135, 2000. 
AZOFRA, V.; PRIETO B.; SANTIDRIÁN, A. The usefulness of a performance measurement system in the daily life of an organisation: a note on a case study. The British Accounting Review, v. 35, n. 4, p. 367-384, 2003.

BALASUBRAMANIAN, S; GUPTA, M. Structural metrics for goal based business process design and evaluation. Business Process Management Journal, v. 11, n. 6, p. 680-694, 2005.

BARBER, N.; SCARCELLI, J. M. Enhancing the assessment of tangible service quality through the creation of a cleanliness measurement scale. Managing Service Quality, v. 20, n. 1, p. 70-88, 2010.

BITITCI, U. S.; CARRIE, A. S.; MCDEVITT, L. Integrated performance e measurement systems: a development guide. International Journal of operations \& production management, v. 17, n. 5, p. 522-534, 1997.

BRUYNE, P. de; HERMAN, J.; SCHOUTHEETE, M. de. Dinâmica da pesquisa em ciências sociais: os pólos da prática metodológica. 5. ed. Rio de Janeiro, RJ: Livraria Francisco Alves, 1991.

BUYTENDIJK, F.; HATCH, T.; MICHELI, P. Scenario-based strategy maps. Business Horizons, v. 53, n. 4, p. 335-347, 2010.

COORDENAÇÃO DE APERFEIÇOAMENTO DE PESSOAL DE NÍVEL SUPERIOR. Portal de Periódicos da CAPES. Disponível em: <http://www.periodicos.capes.gov.br/>. Acesso em: nov. 2012.

CHEN, Mu-Yen; HUANG, Mu-Jung; CHENG, Yu-Chen. Measuring knowledge management performance using a competitive perspective: An empirical study. Expert Systems with Applications, v. 36, n. 4, p. 8449-8459, 2009.

CHIN, Kwai-Sang; LO, Kwong-Chi; LEUNG, Jendy P. F. Development of usersatisfaction-based knowledge management performance measurement system with evidential reasoning approach. Expert Systems with Applications, v. 37, n. 1, p. 366-382, 2010.

DEW, N. et al. Affordable loss: behavioral economic aspects of the plunge decision. Strategic Entrepreneurship Journal, v. 3, n. 2, p. 105-126, 2009.

DEY, P. K. Integrated project evaluation and selection using multiple-attribute decision-making technique. International Journal of Production Economics, $v$. 103, n. 1, p. 90-103, 2006.

DVIR, D.; LECHLER, T. Plans are nothing, changing plans is everything: the impact of changes on project success. Research Policy, v. 33, n. 1, p. 1-15, 2004.

DVIR, D. et al. In search of project classification: a non-universal approach to project success factors Research Policy, v. 27, n. 9, p. 915-935, 1998. 
ENSSLIN, L. et al. Avaliação do desempenho de empresas terceirizadas com o uso da metodologia multicritério de apoio à decisão - construtivista. Pesquisa Operacional, Rio de Janeiro, v. 30, n. 1, 125-152, 2010. Disponível em: <http://www.scielo.br/pdf/pope/v30n1/07.pdf>. Acesso em:

ENSSLIN, S. R. A Incorporação da Perspectiva Sistêmico-Sinérgica na Metodologia MCDA-Construtivista: uma llustração de Implementação. 2002. Tese (Doutorado em Engenharia de Produção)-Programa de Pós-Graduação em Engenharia de Produção, Universidade Federal de Santa Catarina, Florianópolis, 2002.

ENSSLIN, L; ENSSLIN, S. R. Processo de Construção de Indicadores para Avaliação de Desempenho: palestra apresentada ao V Ciclo de Debates: Avaliação de Políticas Públicas. Florianópolis. 2009. Notas prévias.

ENSSLIN, L; ENSSLIN, S. R. Revisão Sistêmica da Literatura. Florianópolis, 1. trim. 2010. Material didático apresentado na disciplina de Avaliação de Desempenho do Programa de Pós-Graduação em Engenharia de Produção da Universidade Federal de Santa Catarina.

ENSSLIN, L. et al. Identificando e analisando problemas de performance: o uso da avaliação de desempenho (feedback, coaching e counseling) para melhorar a produtividade dos empregados. In: Florianópolis: UFSC, 1. sem. 2007. Avaliação de Desempenho.

FOSS, N. J. Bounded rationality and tacit knowledge in the organizational capabilities approach: an assessment and a re-evaluation. Industrial and Corporate Change, v. 12, n. 2, p. 185-201, 2003.

GIBBONS, R. Team theory, garbage cans and real organizations: some history and prospects of economic research on decision-making in organizations. Industrial and Corporate Change, v. 12, n. 4, p. 753-787, 2003.

HSU, Yueh-Ling; LI, Wen-Chin; CHEN, Kuang-Wei. Structuring critical success factors of airline safety management system using a hybrid model. Transportation Research Part E: Logistics and Transportation Review, v. 46, n. 2, p. 222-235, 2009.

HU, Hsiu-Yuan. et al. Using BPNN and DEMATEL to modify importanceperformance analysis model - A study of the computer industry. Expert Systems with Applications, v. 36, n. 6, p. 9969-9979, 2009.

JANSSEN, R. et al. Decision support for integrated wetland management. Environmental Modelling \& Software, v. 20, n. 2, p. 215-229, 2005.

JOINSON, A. N. Self-disclosure in computer-mediated communication: The role of self-awareness and visual anonymity. European Journal of Social Psychology, v. 31, n. 2, p. 177-192, 2001. 
KAPLAN, R. S.; NORTON, D. P. A estratégia em ação: balanced scorecard. Rio de Janeiro: Campus, 1997

KEENEY, R. L. Value focused-thinking: a path to creative decisionmaking. Cambridge: Harvard University Press, 1992.

KETOKIVI, M. A.; SCHROEDER, R. G. Perceptual measures of performance: fact or fiction? Journal of Operations Management, v. 22, n. 3, p. 247-264, 2004.

LACERDA, R. T. de O.; ENSSLIN, L; ENSSLIN, S. R. A Performance Measurement View Of IT Project Management. The International Journal of Productivity and Performance Management, v. 60, n. 2, p. 132-151, 2011.

LEE, C.; LEE, K.; PENNINGS, J. M. Internal capabilities, external networks, and performance: a study on technology-based ventures. Strategic Management Journal, v. 22, n. 6-7, p. 615-640, 2001.

LESKINEN, P. Measurement scales and scale independence in the analytic hierarchy process. Journal of Multi-Criteria Decision Analysis, v. 9, n. 4, p. 163$174,2000$.

LI, Y.; LIAO, X. Decision support for risk analysis on dynamic alliance. Decision Support Systems, v. 42, n. 4, p. 2043-2059, 2007.

MOHAMADABADI, H.S.; TICHKOWSKY,G.; KUMAR, A. "Development of a multicriteria assessment model for ranking of renewable and non-renewable transportation fuel vehicles." Energy, v. 34, n, 1, p. 112-125, 2009.

MCEVILY, S. K.; CHAKRAVARTHY, B. The persistence of knowledge-based advantage: an empirical test for product performance and technological knowledge. Strategic Management Journal, v. 23, n. 4, p. 285-305, 2002.

MITCHELL, R.; BOYLE, B. Knowledge creation measurement methods. Journal of Knowledge Management, v. 14, n. 1, p. 67-82, 2010.

MOHAMADABADI, H. S.; TICHKOWSKY, G.; KUMAR, A. Development of a multicriteria assessment model for ranking of renewable and non-renewable transportation fuel vehicles. Energy, v. 34, n. 1, p. 112-125, 2009.

NEELY, A.; ADAMS, C.; KENNERLEY, M. The performance prism: the scorecard for measuring and managing business success. London: Prentice Hall; Pearson, 2002.

OLSMATS, C.; DOMINIC, C. Packaging scorecard - a packaging performance evaluation method. Packaging Technology and Science, v. 16, n. 1, p. 9-14, 2003 
ÖNÜT, S.; KARA, S. S.; IŞIK, E. Long term supplier selection using a combined fuzzy MCDM approach: A case study for a telecommunication company. Expert Systems with Applications, v. 36, n. 2/part 2, p. 3887-3895, 2009.

PETRI, S. M. Modelo para apoiar a avaliação das abordagens de gestão de desempenho e sugerir aperfeiçoamentos: sob a ótica construtivista. 2005. $236 \mathrm{f}$. Tese (Doutorado em Engenharia de Produção)-Programa de Pós-Graduação em Engenharia de Produção, Universidade Federal de Santa Catarina, Florianópolis, 2005.

RICHARDSON, R. J. et al. Pesquisa social: métodos e técnicas. 3. ed. São Paulo: Atlas, 2008.

ROSA, F. S. Evidenciação Ambiental para a Gestão Interna (EAGI): um instrumento multicritério de apoio à decisão construtivista para a gestão das informações ambientais da empresa Eletrosul S.A. Florianópolis, 2009.

ROSA, F. S. da; ENSSLIN, S. R.; ENSSLIN, L. Evidenciação Ambiental: Processo Estruturado de Revisão de Literatura sobre Avaliação de Desempenho da Evidenciação Ambiental. Sociedade, Contabilidade e Gestão, Rio de Janeiro. v. 4, n. 2, 2009.

ROUWETTE, E. A. J. A.; VENNIX, J. A. M.; MULLEKOM, T. v. Group model building effectiveness: a review of assessment studies. System Dynamics Review, v. 18, n. 1, p. 5-45, 2002.

SELDEN, S. C.; SOWA, J. E. Testing a Multi-Dimensional Model of Organizational Performance: Prospects and Problems. Journal of Public Administration Research and Theory. v. 14, n. 3, p. 395-416, 2004.

SELLTIZ, C. et al. In: MALUFE, J. R.; GATTI, B. (Coords.). Métodos de pesquisa nas relações sociais. 2. ed. São Paulo: Editora Pedagógica e Universitária, 1987.

SHENHAR, A. J. Strategic Project Leadership Toward a strategic approach to project management. R\&D Management, v. 34, n. 5, p. 569-578, 2004.

SHENHAR, A. J.; DVIR, D. Project management research - the challenge and opportunity. Project Management Journal, v. 38, n. 2, p. 93-99, 2007.

SHENHAR, A. J. at al. Refining the search for project success factors: a multivariate, typological approach. R\&D Management, v. 32, n. 2, p. 111-126, 2002.

TASCA, J. E. et al. An approach for selecting a theoretical framework for the evaluation of training programs. Journal of European Industrial Training, v. 34, n. 7, p. 631-655, 2010. 
TUKEL, O. I.; ROM, W. O. An empirical investigation of project evaluation criteria. International Journal of Operations \& Production Management, v. 21, n. 3, p. 400-416, 2001.

VANTI, N. A. P. Da bibliometria à webometria: uma exploração conceitual dos mecanismos utilizados para medir o registro da informação e a difusão do conhecimento. Ci. Inf., Brasília, v. 31, n. 2, p. 152-162, maio/ago. 2002.

WILLIAMS, T. Assessing and moving on from the dominant project management discourse in the light of project overruns. IEEE Transactions on Engineering Management, v. 52, n. 4, p. 497-508, 2005.

WOUTERS, M.; WILDEROM, C.. Developing performance-measurement systems as enabling formalization: A longitudinal field study of a logistics department. Accounting, Organizations and Society, v. 33, n. 4-5, p. 488-516, 2008.

WORREN, N.; MOORE, K.; CARDONA, P. Modularity, strategic flexibility, and firm performance: a study of the home appliance industry. Strategic Management Journal, v. 23, n. 12, p. 1123-1140, 2002.

YLI-RENKO, Helena; AUTIO, Erkko; SAPIENZA, H. J. Social capital, knowledge acquisition, and knowledge exploitation in young technology-based firms. Strategic Management Journal, v. 22, n. 6-7, p. 587-613, 2001.

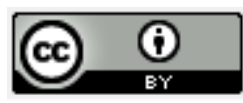

Artigo recebido em 25/09/2010 e aceito para publicação em 16/12/2012. 